\title{
L'animal de rencontre dans la tradition orale algonquienne
}

Richard LEFEBVRE, Collège d'enseignement général et professionnel de l'AbitibiTémiscamingue

\section{L'animal de rencontre}

$\mathrm{Au}$ début du vingtième siècle, Thomas Tailbot Waterman a pu établir, dans une monumentale analyse des corpus de mythes et de contes recueillis par les ethnologues auprès de vingt-trois nations autochtones de l'hémisphère nord-américain, que l'animal est le personnage le plus fréquent dans les récits de la tradition orale $(17,19)$. Les récits de la tradition orale algonquienne ${ }^{1}$, où l'animal est un personnage familier, ne font pas exception. L'animal indigène ${ }^{2}$ partage l'univers diégétique avec l'espèce humaine et les monstres dans un continuum déconcertant en regard des démarcations qui séparent généralement l'homme et l'animal dans les conceptions du monde occidentales. Le personnage de l'animal indigène se retrouve dans des types de situations variées ${ }^{3}$. Je m'intéresse dans cet article à un type de récits dans lequel l'animal est un personnage de rencontre. J'appelle «personnages de rencontre » les personnages secondaires fugaces qui se retrouvent de manière fortuite à des points d'intersection du déplacement spatiotemporel d'un héros déambulant. Bien que cette catégorisation puisse paraître insolite, ce genre de situation et le personnage de second plan qui y est associé sont fréquents dans les cycles narratifs du héros décepteur (trickster) de l'aire de diffusion algonquienne, caractérisée par le mode de vie traditionnel nomade 4 .

Je ne présente ici que très brièvement la nature du héros décepteur, puisque je m'intéresse principalement au personnage secondaire qu'est l'animal de rencontre. L'errance est un trait générique de ce héros. Aiguillonné par la faim, mais très souvent aussi par le désir d'accouplement, ou encore par l'appétit de se faire beau (Boas 42-43), il déambule dans la forêt, et rencontre une variété de personnages, dont la majorité sont des animaux indigènes. Frank Speck rapporte ce commentaire d'un conteur algonquin au sujet du caractère de Wiske'djak ${ }^{5}$ : «He had no canoe, so he always walked about, going great distances here and there, everywhere, until he had travelled almost all over the world»(21). Le révérend Edward Ahenakew trace un portrait similaire de Wesakaychak : «Wesakaychak, was [...] leading what was practically the life of a vagabond. [...] In the course of his travels, he was often hungry » (330). C'est dans le 
contexte de ses déplacements sans fin que le décepteur croise sur son chemin les divers animaux indigènes auxquels je m'intéresserai ici 6 .

La question que j'explorerai dans la suite de cet article est l'une des interrogations que soulève la présence du personnage animal dans la littérature humaine, à savoir : sa représentation dans les scènes de rencontre des récits de la tradition orale algonquienne est-elle une image spéculaire de l'homme, c'est-à-dire une représentation anthropomorphique qui efface les facultés et volontés animales, ou bien correspond-elle à la physiologie, l'éthologie et l'écologie de l'animal indigène?

Le corpus ethnographique que j'ai analysé propose plusieurs épisodes où les personnages animaux se retrouvent inopinément sur la route d'un héros décepteur. Il comprend les collections de récits recueillis et traduits en français par Rémi Savard (1971, 1979), ainsi que les collections de récits colligés et traduits en anglais durant la première moitié du vingtième siècle par Speck (1915), Alanson Skinner (1919), D.S. Davidson (1928), Edward Ahenakew (1929), John T. MacPherson (c. 1930) et Horace P. Beck (1947). Je n’ignore pas que les récits transcrits, traduits et édités par les anthropologues comportent de multiples problèmes pour l'analyse. Craig Womack a mis en lumière les biais qui frappent le corpus documentaire ethnographique : l'effacement des différences tribales, la décontextualisation historique, la dépolitisation des récits, leur reclassement thématique et l'élagage de la non-fiction (Red on Red). Je pense toutefois que l'analyse que j'offre ici, centrée sur la poétique orale et la morphologie du récit, n'est pas trop affectée par ces biais.

\section{Une créature de la poétique orale}

Le patron qui configure l'occurrence de l'animal dans les cycles du décepteur permet de présumer que la narration est déterminée en partie par la poétique de composition orale. Pour saisir le sens de cette thèse, il est nécessaire de comprendre l'architecture des cycles narratifs du héros décepteur et la structure de l'épisode dans lequel apparaît l'animal de rencontre ${ }^{7}$. Ces cycles sont constitués par la juxtaposition d'aventures sans lien de causalité entre eux ${ }^{8}$. Cette architecture des récits du décepteur nord-américain révèle un chronotope similaire à celui du récit d'aventures décrit par Mikhaill Bakhtine, composé de brefs segments ou épisodes dont la série quasi-infinie est gouvernée par le hasard. Celui-ci écrivait, à propos du roman d'aventures grec: «Les aventures elles-mêmes s'enfilent les unes dans les autres dans une série extra- 
temporelle et, en somme, infinie [qui] ne comporte aucune restriction interne importante » (243246). Bakhtine, qui écrivait ceci à la fin des années 1930, avant que ne soient diffusées les observations de terrain de Milman Parry sur les épopées orales slaves et que ne soit formulée la théorie de l'oralité qui allait en résulter, n'a pas remarqué alors le lien entre cette structure du temps de l'aventure et la grammaire narrative du récit épique oral.

Les techniques de composition mises en œuvre durant la performance orale permettent au conteur de surmonter le double défi de produire, à une cadence régulière, devant un auditoire imprévisible, des chaînes d'énoncés et des chaînes d'actions fictives. Dans les cycles narratifs du décepteur où entrent en scène les animaux, les énoncés formulaïques d'ouverture et de fermeture des épisodes, ainsi que les motifs narratifs déclenchant et dénouant les aventures peuvent être interprétés comme des procédés permettant au conteur de générer les énoncés les uns à la suite des autres et de juxtaposer facilement chaque épisode, sans que toute son attention soit occupée par les problèmes d'invention et de cohésion.

Dans la formule d'ouverture des épisodes, se combinent une référence à la situation initiale du héros décepteur placé dans la fonction grammaticale de sujet (ci-après désigné PROTAGONISTE) et un prédicat exprimant le mouvement (ci-après désigné MOUVEMENT). Un marqueur (ci-après désigné MARQUEUR) inséré dans la formule d'ouverture assure ponctuellement la cohésion métanarrative du cycle. Les éléments métanarratifs permettent d'unir les différents épisodes dans une longue performance, ou encore de suspendre la performance à la fin d'un épisode afin de la reprendre à un autre moment. Ces ingrédients formulaïques se retrouvent de manière typique dans l'énoncé d'ouverture d'un épisode du cycle de Carcajou rapporté par Savard : «Carcajou ${ }^{\text {PROTAGONISTE }}$ se mit à errer MOUVEMENT de nouveau MARQUEUR ${ }^{9}$.

À la suite de la formule d'ouverture, le thème de l'aventure est déclenché par un évènement qui introduit le personnage de rencontre, exprimé lui aussi dans un style formulaïque, que j'appellerai, pour le distinguer de la formule d'ouverture qui pose la situation initiale, la formule d'ouverture thématique. Cette formule thématique - qui joue aussi le rôle de l'élément déclencheur dans le développement de l'aventure, ou thème - est composée essentiellement de deux éléments : un prédicat qui exprime l'action d'une rencontre (ci-après désigné RENCONTRE) et la référence au personnage de rencontre placé dans la fonction grammaticale d'objet (ci-après désigné DEUTÉRAGONISTE), le plus souvent un animal, mais pas uniquement ${ }^{10}$. La formule 
d'ouverture et la formule thématique sont enchaînées de manière typique dans l'épisode de la rencontre de Carcajou avec un Ours rapporté par Savard :

Carcajou PROTAGONISTE poursuivit sa route MOUVEMENT et aperçut ${ }^{\text {RENCONTRE }}$ un ours ANTAGONISTE ( Carcajou, 68)

Selon un principe d'extensibilité caractéristique de la poétique orale, les conteurs ont la possibilité d'enrichir les formules sans modifier les contenus essentiels, comme l'illustre le début d'épisode suivant rapporté par Speck :

He [Wiske'djak] ${ }^{\text {PROTAGONISTE }}$ wandered MOUVEMENT across swamps and mountains and around lakes [...]. All at once he PROTAGONISTE came upon RENCONTRE a little flock of partridges newly hatched ANTAGONISTE [...]. (12)

À l'autre extrémité de l'aventure, la formule de clôture de l'épisode varie aussi peu que l'ouverture, bien que l'analyse révèle que l'énoncé formulaïque de clôture est facultatif. Quand elle est présente dans le récit, la formule de clôture suit immédiatement le motif du dénouement (ci-après désigné DÉNOUEMENT) et forme le connecteur de l'épisode en cours avec l'épisode suivant. Voici, par exemple, les versets qui mettent fin à une aventure malheureuse du cycle innu de Mesh rapporté par Savard :

Mesh n'avait plus rien à manger DÉNOUEMENT. Il PROTAGONISTE reprit la route MOUVEMENT. (Contes indiens, 28)

Cette identité entre les formules d'ouverture et de clôture a conduit Andrew Wiget à parler de l'indifférenciation des interfaces de chaque épisode dans les cycles du décepteur (47, 51-52). Quant au motif narratif du dénouement de l'aventure, il est aussi varié que les thèmes qui forment les aventures, mais il a généralement pour fonction de précipiter le héros décepteur dans une situation finale identique à la situation initiale de l'épisode. Le telos à peu près invariable des aventures est le nord vers lequel se tourne la boussole du compositeur lorsqu'il produit la chaîne d'actions d'un épisode, permettant ainsi la juxtaposition des aventures dans le cycle narratif. En somme, les formules d'ouverture et de fermeture, ainsi que les motifs de déclenchement et de dénouement simplifient la composition des épisodes et du cycle.

Ce que laisse entendre l'existence de ce patron narratif, c'est que le thème et la sphère d'action des personnages animaux sont subordonnés en partie aux impératifs techniques de la composition orale. L'animal indigène est, à cet égard, une créature de la poétique des récits algonquiens, poétique dont les contraintes restreignent l'expression de son éthogramme. 


\section{Le décepteur déçu}

Les thèmes des épisodes de rencontre à l'intérieur d'un même cycle ou dans des cycles éloignés ont un air de famille qui fait dire aux lecteurs profanes qui s'aventurent dans le corpus ethnographique des récits algonquiens que les histoires se répètent. Cette observation se vérifie sur deux plans. D'une part, bien qu'elles expriment un éventail de thèmes dont la texture varie au gré de la substitution des accessoires et des espèces, les rencontres du décepteur avec un animal n'excèdent pas un nombre limité de thèmes ${ }^{11}$. D'autre part, indépendamment des thèmes, les épisodes se développent de façon assez semblable. La situation initiale dans laquelle se trouve le décepteur est source de déséquilibre. La rencontre inopinée avec l'animal déclenche donc une première séquence de motifs, que j'appelle séquence ascendante, où l'animal - ou tout autre personnage secondaire - a pour fonction de supprimer le déséquilibre initial. Le développement de l'épisode est ensuite marqué par une péripétie - ou par une cascade de péripéties dans les épisodes plus complexes - qui opère un renversement, annule le gain de la première séquence d'actions et retourne le héros dans une situation finale problématique. Dans cette nouvelle séquence de l'épisode, que je qualifie de séquence descendante, de nouveaux personnages interviennent : quelquefois des hommes (des «Indiens »), le plus souvent des animaux. Voici un bref exemple de l'enchaînement de cette double séquence dans un récit que rapporte de manière sommaire Beck :

One time Wisekedjak killed a moose with a stone on the side of a mountain. He began to cook some of the meat and dry the rest [SÉQUENCE ASCENDANTE], when he heard a noise. Thinking it was a bird, he climbed a tree to find out. It was the wind, but Wisekedjak couldn't get down. While he was in the tree, a big wolf came and ate all the moose ${ }^{[S E ́ Q U E N C E ~ D E S C E N D A N T E]} .(263)$

Il est possible de reconnaître, dans ce passage, le motif traditionnel de l'arbre piège (tree snare). Il est également possible d'observer la concaténation des séquences ascendantes et descendantes. Cette construction qui trouve dans l'extrait de Beck une expression sommaire, peut être enrichie par une multiplication de motifs intermédiaires, comme c'est le cas dans l'épisode suivant d'un récit algonquin rapporté par Speck. Au début de cet épisode, Wiske'djak est affamé et cherche quelque chose à manger. Il rencontre sur son chemin un Ours qu'il embobine, assomme, dépèce et met à cuire. C'est la séquence ascendante, qui satisfait la quête du héros. Au moment d'entamer son festin cependant, Wiske'djak est agacé par le bruit que fait le vent dans les 
branches. Il monte alors dans l'arbre où sa main reste prisonnière des branches. C'est le motif de l'arbre piège, qui constitue le point tournant de l'épisode. «Pretty soon » poursuit le narrateur, when he [Wiske'djak] looked down, he saw a Squirrel come and take some of his meat. He shouted for him to go away without any success. Next came the Marten, then the Fisher, then some Wildcats, then Ravens, and in fact all kinds of animals came and began to eat up his supply of meat. He tried to drive them away, but couldn't. The more he shouted at them, the more they danced and sang and ran off mocking him. They carried away all his pieces of meat to their dens. (5)

À la suite de cette razzia qui initie la séquence descendante, le héros décepteur voit son panier de graisse d'ours dispersé dans la rivière par Rat Musqué, puis le bouillon resté sur le feu subtilisé par Carcajou. À la fin, Wiske'djak se retrouve emprisonné dans le crâne de l'animal alors qu'il tente d'en manger la cervelle, et il est pourchassé par les Indiens qui le prennent pour un ours. Au bout de cette série de malheurs, Wiske'djak se retrouve dans une situation semblable à la situation initiale ${ }^{12}$. Une fois de plus, l'analyse conduit à l'observation d'un patron narratif qui détermine en partie la sphère d'action du personnage animal.

Le cycle des séquences ascendantes et descendantes qui charpentent la plupart des épisodes de rencontre du décepteur avec les animaux a été thématisé par certains conteurs, et signalé par plusieurs anthropologues. Savard rapporte, en traduction, une curieuse expression qui exprime un tel renversement, alors que deux femmes se rendent auprès de Carcajou afin d' « excréter sur l'excréteur » (Carcajou, 42). Mac Linscott Ricketts dit du décepteur : «He is [...] a blunderer who is often the victim of his own tricks and follies » (327). La fable du décepteur déçu introduit une conception dynamique de la place de l'homme et de l'animal dans le cosmos. Dans les séquences narratives ascendantes et descendantes, qui correspondent à des cycles de prédation et de déprédation, les personnages animaux et les hommes peuvent occuper, si l'on adopte l'interprétation selon laquelle le héros décepteur est une incarnation mythique de l'homme, deux positions opposées - celle de la proie et celle du déprédateur - ou se retrouvent tous deux dans le même paradigme du déprédateur et peuvent être substitués. Cette mobilité des catégories d'acteurs dans le schéma actanciel révèle une conception algonquienne des relations extraspécifiques qui s'écartent des représentations les plus prégnantes du rapport entre l'homme et l'animal dans les récits de la tradition occidentale, notamment celle de la chaîne alimentaire qui adopte la forme idéologique de la pyramide. En somme, le patron qui charpente de manière générale les épisodes et semble à première vue modeler la représentation des interactions entre 
les êtres vivants introduit également une conception dynamique de la place de chaque espèce dans son milieu.

\section{Dialogue et prosopopée}

La présence habituelle d'interactions verbales dans les épisodes de rencontre et dans les mythes en général indique l'importance du régime discursif dialogal dans l'art du récit oral algonquien. Il ressort de l'analyse des scènes dialoguées ceci : le patron narratif qui s'impose sur le plan des énoncés et de la construction des épisodes, prévaut jusque dans l'orientation des actions réalisées dans les interactions verbales.

Le dialogue survient très tôt dans l'épisode de rencontre avec l'animal. Il commence par l'interpellation de l'animal : «Kwe, Muskrat! (Speck 6). L'interpellation allègue souvent une appartenance sociale : «Nista'tuk», «Jeunes frères », «Jeunes sœurs » (Savard, Carcajou, 24, 28, 39), «Mon jeune frère », «Mon petit frère » (Contes indiens, 27, 29), «Nicim », «my younger brother »(Skinner 280, 288), «my brother » (Ahenakew 337). Grâce au pouvoir de la parole de voyager entre la bouche et l'oreille sur une distance plus grande que la portée de la main, l'interpellation réduit l'intervalle entre le héros déambulant et l'animal qu'il croise inopinément. Interpelés, les animaux répondent à la parole qui leur est adressée et s'engagent dans un dialogue.

Le dialogue avec l'animal est un expédient par lequel le protagoniste poursuit la satisfaction de sa quête. L'analyse des transactions dans les interactions verbales des séquences ascendantes montre que, dans les dialogues structurellement simples, les questions (ou les requêtes, ci-après désignées par $\alpha$ ) sont suivies par les réponses (ou le consentement, ci-après désignés par $\beta$ ) dans des paires à peu près adjacentes. La rencontre de Wiske'djak avec l'Ours rapportée par Speck offre un exemple d'une transaction structurellement simple orientée vers la satisfaction de la quête du héros décepteur :

[Wiske'djak] went up to the bear and said : «Hello, Bear ! What's the matter with you ? » ${ }^{[\alpha 1]}$ The Bear answered, «Oh! my eyes are so sore, and I can't see where to go ${ }^{[\beta 1]}$. I just wish somebody would help me. ${ }^{[\alpha 2]}{ }_{\gg}$ «Well, come along with me $\left[{ }^{\left[3^{3}\right]}\right.$. I will help you ${ }^{[\beta 2]}$. I know where to get some fine medecine that will fix you up all right ». «Very well, » said the bear ${ }^{[\beta 3]}$. (Speck 4)

Dans les séquences descendantes, le sens des transactions est inversé : les requêtes ou les questions du protagoniste sont rejetées ou obtiennent une réponse négative (ci-après désigné par 
$\omega)$. Dans le corpus que j'ai analysé, l'exemple le plus simple pour illustrer cette inversion des transactions ne se trouve pas dans un dialogue du décepteur avec un animal, mais dans une reprise du motif de l'arbre piège rapportée par Speck. Dans ce dialogue en style direct, Wiske'djak adresse la parole à un arbre :

Said Wiske'djak, « you had better stop that noise until I get through eating. I don't like it at all » ${ }^{[\alpha 1]} \ll \mathrm{Oh} ! »$ said the Tree, « I have to do it. I can't stop it. » ${ }^{[\omega 1]}$

À la suite de cette réponse négative, Wiske'djak grimpe dans l'arbre et ses mains se prennent entre les branches.

«Let me go ! Let me go ! ${ }^{\left[\alpha{ }^{2}\right]}$ » he [Wiskedjak] begged to the tree. «I must get down to my meat. » But the only answer he got was, «No » ${ }^{[\omega 2]}$, and there he stayed. (5)

Ce que ces exemples mettent en évidence, c'est que l'orientation de l'action réalisée à travers les interactions verbales est déterminée par leur position dans la chaîne des séquences. Les mêmes orientations sont observables dans des systèmes d'interaction structurellement plus complexes qui sont bien entendu plus riches en rebondissements et plus comiques.

Le dialogue introduit une figure familière de la littérature animalière, la prosopopée, par laquelle un langage humain est attribué à l'animal. Comment doit-on interpréter cette représentation ? Faut-il considérer la prosopopée comme un anthropomorphisme niant, usurpant, ventriloquant, transformant l'animal en métaphore ou allégorie du devenir ou de l'essence humaine, comme elle a été généralement considérée par les critiques (cf. Armbruster 17, 20-23 ; Haraway 20 ; McHugh 7) ?

Les composantes essentielles du mécanisme de l'anthropomorphisme que sont les catégories de l'homme et de l'animal, ne sont pas aussi nettement distinctes ou opposées dans la conception algonquienne du monde, telle qu'elle se laisse voir dans les épisodes de rencontre et autres récits de la tradition orale où interviennent des personnages animaux. J'ai signalé dans une note de cet article que l'animal n'est pas la seule catégorie de personnage à occuper la position du deutéragoniste. J'ai fait remarquer que celui-ci relève d'une catégorie plus large qui inclut, entre autres, des minéraux, des végétaux et des humains. Dans une autre note, j'ai mentionné que le décepteur revêt parfois des formes animales, parfois la forme humaine. Les commentaires par lesquels les conteurs expliquent, justifient ou motivent ces paradoxes éclairent cette conception algonquienne. Un commentaire narratif recueilli par Speck affirme que, dans le passé mythique, 
les animaux parlaient entre eux : «All animals could then talk together » (48). Dans sa collection de récits mettant en scène le décepteur Wesakaychak, Ahenakew explique : «In these old narratives, there is only a slight demarcation between what is human and what is animal » (323). Plusieurs commentaires précisent qu'il existe des êtres, comme le décepteur (mais pas seulement lui), qui ont le pouvoir de faire parler les animaux, mais aussi les arbres et les pierres. Speck rapporte cette glose à propos du héros Wiske'djak : «He had the power to make everything in creation answer him when he spoke to it ; trees, water, animals, and all the other little creatures would reply to him when he spoke » (21). Lorsque, dans un épisode du cycle de Mesh, celui-ci n'obtient pas de réponse en interpelant une énorme pierre, il répète sa demande jusqu'à ce que la pierre réponde enfin (Savard, Contes indiens, 30). D'autres commentaires soutiennent que le décepteur est capable de parler dans la langue des autres. Dans un épisode rapporté par Savard où Carcajou s'adresse à une pierre, le conteur commente l'action en expliquant que « Carcajou peut parler à n'importe quoi »(Carcajou, 65). Davidson rapporte cette précision recueillie au Grand Lac Victoria à propos du personnage de Meso : « He was able to talk the language of all things, animate and inanimate as well » (278).

Il est évident que les scènes dialoguées dans les récits algonquiens s'inscrivent dans une conception du monde qui diverge, par exemple, des thèses anthropocentriques heideggériennes selon lesquelles - rapporte Jacques Derrida - l'homme est configurateur du monde, alors que la pierre est sans monde, et l'animal pauvre en monde (210). En somme, l'animal parlant et la prosopopée ne présentent pas, dans le récit algonquien où il n'y a pas d'opposition constitutive entre l'homme et l'animal, le problème qu'ils posent dans la littérature humaniste et la tradition philosophique occidentale où il y a une démarcation nette entre $1^{\prime}$ homme et l'animal ${ }^{13}$.

\section{Le langage de la performance}

Tout comme les genres littéraires, les récits de la tradition orale algonquienne sont unis par un principe d'identité qui conjure dans une certaine mesure l'aléatoire de l'invention, pour reprendre les termes de Michel Foucault dans L'ordre du discours à propos des procédures de réglage de la production discursive. Les épisodes de rencontre avec l'animal ne sont jamais absolument nouveaux : ils sont conditionnés par l'horizon culturel que partagent les poètes du récit oral et les auditeurs. L'intertexte oral se manifeste dans les motifs et les énoncés formulaïques qui véhiculent des réminiscences du genre. Aussi divers soient-ils, les personnages 
animaux se glissent dans des fonctions actancielles limitées qui effacent en partie les différences spécifiques entre les espèces. L'incidence de la poétique orale et de la tradition narrative sur la représentation de l'animal dans le récit est une conclusion qui ne fait aucun doute.

En outre, la sémiotique de la communication animale - c'est-à-dire le langage au moyen duquel les créatures vivantes communiquent (Armbruster 27$)^{14}$ - semble absente des scènes de rencontre qu'offrent les textes du corpus ethnographique. Mais il faut questionner la capacité des textes à enregistrer les modes de communication qui n'ont pas la forme verbale du langage humain. La transcription ethnographique efface plusieurs des contenus de la performance qui est à la source du texte. La performance orale offre en effet un potentiel d'expression varié, qui peut être communiqué à travers le canal vocal (changements de voix, débit, intensité) et à travers le canal visuel (regards, mimiques, gestes, déplacements). Ce sont ces canaux qui sont effacés dans la transcription en raison des limites de l'écriture à en reproduire les signes. La performance orale met donc en œuvre un système de signification complexe qui va au-delà de la sémiotique littéraire qui a animé dans cet article les analyses des récits algonquiens. Ce système sémiotique syncrétique, qui met à contribution non seulement la langue, mais la voix et tous les organes du corps capables d'émettre des signes, est apparenté à la communication corporelle au moyen de laquelle les créatures vivantes échangent entre elles.

Dans un article où elle a exploré l'art performatif traditionnel amérindien, Geraldine Manossa a raconté l'expérience passée d'une performance orale où elle se trouvait dans l'auditoire. Dans cet essai autobiographique, Manossa se retrouve dans la position de la narratrice d'un premier niveau de récit - elle relate, décrit, commente la performance du conteur cri Eddie Bellrose - alors que les paroles d'Eddie racontant une aventure de Wasakaychak constituent un deuxième niveau de récit. La narratrice met en évidence les simagrées, les gesticulations et les silences du conteur, ainsi que les réactions des auditeurs, dont le récit du deuxième niveau - analogue au texte des ethnographes, avec cette différence que le conteur performe un récit qui est propre à sa culture - ne rend évidemment pas compte. Le point de vue de Manossa - qui, elle, partage la culture du conteur - met en lumière les multiples canaux d'expression de la performance. Elle raconte, par exemple : «We anxiously wait for the change in tone and rhythm of Eddie's voice. His voice is the orchestra that dictates the movements of his body. This is how he brings to life all the beings of the story » (173). En tenant compte du potentiel de la performance, que corrobore la narration de Manossa, ne peut-on avancer 
l'hypothèse que la représentation de l'animal dans les récits algonquiens, et en particulier dans les épisodes de rencontre, soit le lieu d'une affirmation de sa singularité, en dehors du mécanisme spéculaire anthropomorphique?

\section{Bibliographie}

Ahenakew, Edward. «Cree Trickster Tales ». The Journal of American Folklore 42.166 (1929) : 309-353.

Armbruster, Karla. «What Do We Want from Talking Animals ? Reflections on Literary Representations of Animal Voices and Minds». Speaking for Animals: Animal Autobiographical Writing. Dir. Margo DeMello. New York : Routledge. 17-33.

Babcock, Barbara. "'A Tolerated Margin of Mess': The Trickster and His Tales Reconsidered». Critical Essays on Native American Literature. Dir. Andrew Wiget. Boston : G.K. Hall, 1985. 153-185.

Bakhtine, Mikhaïl. Esthétique et théorie du roman. Tr. du russe par D. Olivier. Paris : Gallimard, 1987.

Beck, Horace P. «Algonquin Folklore from Maniwaki ». The Journal of American Folklore 60.237 (1947) : 259-264.

Boas, Franz. «Mythology and Folk-Tales of the North American Indians ». Critical Essays on Native American Literature. Dir. Andrew Wiget. Boston : G.K. Hall, 1985. 28-50.

Bouchard, Serge et Josée Mailhot. «Structure du lexique : les animaux indiens ». Recherches amérindiennes au Québec: Signes et langages des Amériques 3.1-2 (1973) : 39-67.

Davidson, D. S. «Tales from Grand Lake Victoria, Quebec ». The Journal of American Folklore 41.160 (1928) : 275-282.

Foucault, Michel. L'ordre du discours. Paris : Gallimard, 1971.

Haraway, Donna J. When Species Meet. Minneapolis : U of Minnesota P (Posthumanities 3), 2008.

Kaye, Jonathan D. et al. Odawa Language Project First Report. $2^{\mathrm{e}}$ éd. Toronto : Dept. of Anthropology (Anthropological Series 9), 1973.

Lefebvre, Madeleine. Tshakapesh : Récits montagnais-naskapi. [S.1] : Éditeur officiel du Québec / Ministère des affaires culturelles (Cultures amérindiennes 4), 1971. 
Manossa, Geraldine. «The Beginning of Cree Performance Culture ». (Ad)dressing Our Words : Aboriginal Perspectives on Aboriginal Literatures. Dir. Armand Garnet Ruffo. Penticton, $2001: 169-180$.

MacPherson, John T. An Ethnological Study of the Abitibi Indians [tapuscrit]. Musée canadien des civilisations : Fonds MacPherson, John T. (III-G-38M), boîte 50 f.1.

McHugh, Susan. Animal Stories : Narrating across Species Lines. Minneapolis : U of Minnesota P, 2011.

Radin, Paul. Winnebago Hero Cycle : A Study in Aboriginal Literature. Baltimore : Waverly P, 1948.

Ricketts, Mac Linscott. «The North American Indian Trickster». History of Religion 5.2 (1966) : 327-350.

Savard, Rémi. Carcajou et le sens du monde : récits montagnais-naskapi. [S.1.] : Éditeur officiel du Québec, 1971.

---. Contes indiens de la Basse Côte Nord du Saint-Laurent. Ottawa: Musées nationaux du Canada, 1979.

Skinner, Alanson. «Plains Ojibwa Tales ». The Journal of American Folklore 32.124 (1919) : 280-305.

Speck, Frank G. Myths and Folk-lore of the Timiskaming Algonquin and Timagami Ojibwa. Ottawa : Government Printing Bureau / Department of mines, 1915.

Waterman, Thomas Tailbot. «The Explanatory Element in the Folk-Tales of the North American Indians ». The Journal of American Folklore 27.103 (1914) : 1-54.

White, Richard. Le Middle Ground: Indiens, empires et républiques dans la région des Grands Lacs, 1650-1815. Tr. de 1'anglais par F. Cotton. Toulouse : Anacharsis, 2009.

Wiget, Andrew. "Cycle Construction and Character Development in Central Algonkian Trickster Tales ». Oral Tradition 15.1 (2000) : 39-73.

Womack, Craig S. Red on Red: Native American Literary Separatism. Minneapolis : U of Minnesota P, 1999.

\section{NOTES}

${ }^{1}$ Une précision terminologique s'impose. J'ai choisi, par commodité, de suivre l'exemple de Richard White qui emploie le terme "Algonquien» (avec la majuscule) pour faire référence à un ensemble de nations nordamérindiennes qui ne parlent pas toutes une langue algique (comme les Winnebagos, un groupe dont il sera question dans cet article, qui parle une langue de la famille linguistique siouane) et qui occupent un territoire qui était autrefois appelé le Pays d'en Haut (White 25-26). Le terme courant «algonquien » (avec la minuscule) réfère à une 
large famille linguistique de l'Amérique du Nord. Le terme «Algonquin » (avec la majuscule) a été utilisé de manière confuse par les chroniqueurs aux seizième et dix-septième siècles pour désigner des groupes de langues algonquiennes de l'Est du Canada, et désigne une nation anishinaabe actuelle du nord-ouest du Québec. Quant au terme «algonquin » (avec la minuscule), il renvoie aux dialectes des communautés autochtones de l'Abitibi et du Témiscamingue (dialectes dits de l'algonquin du Nord) et de Maniwaki (dialecte dit de l'ojibwa de l'Est).

${ }^{2}$ Dans une étude lexicale de la langue innue réalisée par Josée Mailhot et Serge Bouchard intitulée «Structure du lexique: Les animaux indiens », les auteurs signalent qu'un des critères de classification de l'animal (wesis) distingue l' « animal indien » (innuwesis) de l'« animal blanc » (kakusse syuwesis).

${ }^{3}$ Loin de présenter ici une typologie exhaustive, je ne veux que témoigner de la diversité des types de situations dans lesquelles se retrouve le personnage animal dans les récits de la tradition orale algonquienne. Le personnage animal évolue parfois en clan composé d'espèces diverses, comme dans les cycles de Wemicus ou de Cyngaris rapportés par F. G. Speck (17-20, 39-53) ou quelques courts récits d'Abitibi rapportés par John T. Macpherson (243244, 256-259). D'autres récits présentent un animal à la taille gigantesque menaçant dans la plupart des cas l'espèce humaine, comme le Katshitushk (Lefebvre 22-25), le grand Élan (Ahenakew 316), le grand Castor (Speck 2-3; Davidson 277-278), Misamich (Macpherson 136-141), Shegak (Macpherson 206). Je n'ai trouvé qu'un seul récit, rapporté par Glyne Piggot et Jonathan Kaye, où l'animal est un double de l'homme, ou un nagual, une situation fréquente dans la tradition méso-américaine : «A Battle in the War of 1812 » (Kaye, et al. 82-99).

${ }^{4}$ Dans l'étude intitulée "The North American Indian Trickster », Mac Linscott Ricketts fait 1'hypothèse que le décepteur est une figure archaïque issue de la culture des cueilleurs-chasseurs (328).

${ }^{5}$ Le décepteur est un archétype. Il est incarné dans les cultures locales par différentes figures dont le nom est orthographié de multiples façons dans les textes ethnographiques. J'ai choisi de reproduire la graphie des noms propres telle que je la trouve dans chacune des sources textuelles dont il est question.

${ }^{6}$ Le décepteur exhibe occasionnellement un caractère anthropomorphique, que Paul Radin avait remarqué dans l'introduction du mémoire sur la figure du décepteur winnebago qu'il a publié en 1948 : «He [le décepteur] behaves like a human being in a human environment »(17). Dans les récits algonquiens, ce caractère humain implicite se manifeste dans son utilisation des accessoires (panier, couteau, sac, violon, wigwam, etc.) et les savoir-faire (notamment l'utilisation du feu et des techniques de cuisson) de la culture humaine. Selon un informateur de John T. MacPherson, la capacité du décepteur d'adopter la forme humaine fait partie de ses multiples pouvoirs: «Whiskeyjack had the power of turning himself into human form » (192). Dans certains récits, le décepteur revêt des formes animales, notamment celle du serpent (Skinner 289 ; Beck 263). Le personnage du décepteur prenant part au façonnement du monde dans certains mythes est identifié à un démiurge (trickster-transformer) ; le décepteur apportant aux communautés humaines les savoir-faire qui contribuent à leur conservation et mieux-être est désigné comme un héros culturel.

${ }^{7}$ Plusieurs chercheurs qui ont étudié les cycles narratifs du décepteur nord-américain ont attiré l'attention sur des formes de composition orale dans les transcriptions, notamment Barbara Babcock dans « 'A Tolerated Margin of Mess': The Trickster and His Tales Reconsidered» (167). Andrew Wiget offre une analyse extensive de cette question dans «Cycle Construction and Character Development in Central Algonkian Trickster Tales ».

8 «The creation of links beween episodes is associative, not causal » (Wiget 59).

${ }^{9}$ (Savard, Carcajou 64). Les indices identifiant les éléments formulaïques ont été, bien entendu, ajoutés par moi.

${ }^{10} \mathrm{~L}$ 'animal n'est pas la seule catégorie à tenir le rôle de personnage secondaire dans l'épisode de rencontre type. On trouve aussi des personnages qui relèvent du monde minéral (Davidson 276-277, 278) ou végétal (Speck 10,12 ; Skinner 289). Je reviens sur cette question dans la conclusion de l'article.

${ }^{11}$ Voir l'inventaire de thèmes des cycles narratifs du décepteur proposé par Andrew Wiget (Wiget 54-55).

12 Je n'ai présenté ici, dans le but d'en rendre la fonction plus claire, qu'un seul motif de dénouement, soit celui de la subtilisation de la nourriture du décepteur par un personnage tiers (homme ou animal). Ce motif est parfois remplacé par d'autres motifs, comme par exemple le motif explicatif, reconnu au début du siècle par Waterman («The Explanatory Element in the Folk-Tales of the North American Indians »). C'est ce motif qui clôt la plupart des épisodes du cycle de Wesakaychak rapporté par Edward Ahenakew. Un autre motif est celui par lequel le conteur, adoptant la voix narrative homodiégétique du témoin, invoque son absence momentanée de l'univers diégétique pour justifier son ignorance du dénouement. Ce type de motif termine de nombreux épisodes du cycle algonquin de Wiske'djak rapporté par Frank Speck.

${ }^{13}$ Pour un résumé de l'évolution de la conception de l'animal au sein de la tradition philosophique occidentale, voir «The Speaking Animal Speaking the Animal : Three Turning Points in Thinking the Animal » de Claudia Egerer. Ces conceptions philosophiques ont été longuement examinées et font l'objet d'une entreprise de déconstruction par Jacques Derrida dans L'animal que je donc je suis. 
${ }^{14}$ Donna Haraway emprunte le concept de communication corporelle (embodied communication) de la bioanthropologue Barbara Smuts pour évoquer l'existence d'une danse relationnelle (dance of relating), une sorte de langage du middle ground entre les espèces (25-26). 